\title{
Symmetries in the angular distribution of exclusive semileptonic B decays*
}

\section{Tobias Hurth ${ }^{\dagger}$}

Institute for Physics, Johannes Gutenberg-University, D-55099 Mainz, Germany

E-mail: tobias.hurth@cern.ch

\section{Ulrik Egede}

Imperial College London, London SW7 2AZ, United Kingdom

E-mail: u.egede@imperial.ac.uk

\section{Joaquim Matias}

Universitat Autònoma de Barcelona, 08193 Bellaterra, Barcelona, Spain

E-mail: matias@ifae.es

\section{Marc Ramon}

Universitat Autònoma de Barcelona, 08193 Bellaterra, Barcelona, Spain

E-mail: mramoneifae.es

\section{Will Reece}

CERN, Dept. of Physics, CH-1211 Geneva 23, Switzerland

E-mail: will.reecedcern.ch

We discuss a method to construct observables protected against QCD uncertainties based on the angular distribution of the exclusive $B_{d} \rightarrow K^{* 0}(\rightarrow K \pi) l^{+} l^{-}$decay. We focus on the identification and the interpretation of all the symmetries of the distribution. They constitute a key ingredient to construct a set of so-called transverse observables. We work in the framework of QCD factorization at NLO supplemented by an estimate of power-suppressed $\Lambda / m_{b}$ corrections. A discussion of the new physics properties of two of the transverse asymmetries, $A_{T}^{(2)}$ and $A_{T}^{(5)}$, is presented. A comparison between the transverse asymmetry $A_{T}^{(2)}$ and the forward-backward asymmetry shows that $A_{T}^{(2)}$ emerges as an improved version of it.

The 35th International Conference on High Energy Physics - ICHEP2010, July 21-28 2010, Paris, France

*UAB-FT-686, MZ-TH/10-48

† Speaker. 


\section{Introduction}

The next decade will be hopefully dominated by the new physics discoveries at LHC. In this scenario flavour physics will play an important and complementary role to direct discovery, by exploring the flavour sector of the theory that lies beyond the SM. Rare B decays are sensitive probes to new physics signals (for a recent review see Ref. [1]). Amongst them the semileptonic exclusive decay $B \rightarrow K^{*} l^{+} l^{-}$is particularly important due to its very rich phenomenology. Some of the observables constructed out of this decay are: the forward-backward asymmetry, $A_{F B}$, and its zero [2, 3, 4], the isospin asymmetry, $A_{I},[5,6]$, and the transverse observables $A_{T}^{(i)}(\mathrm{i}=2,3,4,5)$ based on the four-body angular distribution when the $K^{*}$ decays into a $K \pi$ pair [7, 8, 9, 10]. Also the coefficients of the angular distribution [11] or ratios between different $q^{2}$ regions [12] are used to define observables. The main focus of this paper is to provide a guideline for the construction of transverse observables $\left(A_{T}^{(i)}\right)$. These observables maximize the sensitivity to new physics and, at the same time, exhibit a minimal hadronic uncertainty, in particular, to the poorly known soft form factors.

\section{General method}

In this section we will describe the basis of the method recently completed in [10] to construct robust transverse ${ }^{1}$ observables. The method is sufficiently general to be applied to angular distributions with similar properties. The steps of the method are: 1) use the helicity amplitudes of the $K^{*}$ as the key ingredients to construct a quantity where the soft form factor dependence cancels at LO (amplitudes in the large recoil limit are very useful to check this cancellation) 2) identify all symmetries of the distribution with respect to transformations of the $K^{*}$ spin amplitudes 3 ) check that the constructed quantity fulfils all the symmetries to identify it as an observable 4) express the observable in terms of the coefficients of the distribution. As a by-product of the method hidden correlations between the coefficients of the distribution may arise. These correlations have proven to be important for the stability of the fit and also provide a powerful extra experimental check.

Our main source of information is the differential decay distribution of the decay $\bar{B}_{d} \rightarrow \bar{K}^{* 0}(\rightarrow$ $\left.K^{-} \pi^{+}\right) l^{+} l^{-}$with the $K^{* 0}$ on the mass shell. This distribution is a function of four variables

$$
\frac{d^{4} \Gamma}{d q^{2} d \cos \theta_{l} d \cos \theta_{K} d \phi}=\frac{9}{32 \pi} J\left(q^{2}, \theta_{l}, \theta_{K}, \phi\right)
$$

where $q^{2}=s$ is the square of the lepton-pair invariant mass, $\theta_{l}$ is the angle between $\overrightarrow{p_{l^{+}}}$in $l^{+} l^{-}$ rest frame and di-lepton's direction in rest frame of $\bar{B}_{d}, \theta_{K}$ is the angle between $\overrightarrow{p_{K}^{-}}$in the $\bar{K}^{* 0}$ rest frame and direction of the $\bar{K}^{* 0}$ in rest frame of $\bar{B}_{d}$, and finally $\phi$ is the angle between the di-lepton plane and the $K-\pi$ plane. The function $J\left(q^{2}, \theta_{l}, \theta_{K}, \phi\right)$ splits into the following coefficients of the distribution $[10,11]$

$$
\begin{gathered}
J\left(q^{2}, \theta_{l}, \theta_{K}, \phi\right)= \\
J_{1 s} \sin ^{2} \theta_{K}+J_{1 c} \cos ^{2} \theta_{K}+\left(J_{2 s} \sin ^{2} \theta_{K}+J_{2 c} \cos ^{2} \theta_{K}\right) \cos 2 \theta_{l}+J_{3} \sin ^{2} \theta_{K} \sin ^{2} \theta_{l} \cos 2 \phi+
\end{gathered}
$$

\footnotetext{
${ }^{1}$ Indeed only $A_{T}^{(2)}$ and $A_{T}^{(5)}$ are strictly transverse observables, $A_{T}^{(3)}$ and $A_{T}^{(4)}$ being also sensitive to the longitudinal spin amplitudes should, for consistency, be called transverse/longitudinal observables.
} 


$$
\begin{gathered}
+J_{4} \sin 2 \theta_{K} \sin 2 \theta_{l} \cos \phi+J_{5} \sin 2 \theta_{K} \sin \theta_{l} \cos \phi+\left(J_{6 s} \sin ^{2} \theta_{K}+J_{6 c} \cos ^{2} \theta_{K}\right) \cos \theta_{l}+ \\
+J_{7} \sin 2 \theta_{K} \sin \theta_{l} \sin \phi+J_{8} \sin 2 \theta_{K} \sin 2 \theta_{l} \sin \phi+J_{9} \sin ^{2} \theta_{K} \sin ^{2} \theta_{l} \sin 2 \phi
\end{gathered}
$$

These coefficients $J_{i}$ with $i=1 s, 1 c, 2 s, 2 c, 3-5,6 s, 6 c, 7-9$ are in turn functions of the amplitudes $A_{\perp, \|, 0, t, S}^{(L, R)}[10,11]\left(A_{\perp, \|, 0}\right.$ are linear combinations of the well-known helicity amplitudes $\left.H_{+1,-1,0}\right)$. The counting of the coefficients of the angular distribution and of the theoretical spin amplitudes depends on whether scalar interactions are relevant in the analysis or not. If we include them we have 8 complex amplitudes $\left(A_{\perp, \|, 0,(L, R) S, t}\right)$ and 12 experimental inputs $\left(J_{i}\right)$, while if no scalar amplitudes are considered we would have just 7 complex amplitudes $\left(A_{\perp, \|, 0,(L, R), t}\right)$ and 11 experimental coefficients $\left(J_{6 c}=0\right)$. If we neglect the mass of the lepton in addition, the number of complex spin amplitudes gets further reduced to $6\left(A_{t}=0\right)$.

\section{Symmetries of the distribution}

Experimental $\left(J_{i}\right)$ and theoretical $\left(A_{i}\right)$ degrees of freedom have to match. The equation that defines this matching is $n_{C}-n_{d}=2 n_{A}-n_{s}$, where $n_{C}$ is the number of coefficients of the differential distribution $\left(J_{i}\right), n_{d}$ is the number of relations between the $J_{i}, n_{A}$ is the number of spin amplitudes, and $n_{s}$ is the number of symmetries of the distribution.

We will focus here on the case of massless leptons with no scalars. The parameters of the equation are then $n_{C}=11, n_{d}=3\left(J_{1 s}=3 J_{2 s}, J_{1 c}=-J_{2 c}\right.$, and a third more complex relation), $n_{A}=6$ (spin amplitudes), $n_{s}=4$ symmetries. One of the main results in Ref. [10] was to identify the fourth and last symmetry (three of them were found in Ref. [9]). Moreover, a non-trivial hidden correlation between the coefficients of the distribution was discovered.

One important question arises at this point: how do we know that there are four symmetries without having found first the new non-trivial hidden correlation?

In order to count the number of symmetries we define an infinitesimal symmetry transformation of the distribution: $\overrightarrow{A^{\prime}}=\vec{A}+\delta \vec{S}$ where

$$
\begin{aligned}
\vec{A}= & \left(\operatorname{Re}\left(A_{\perp}^{L}\right), \operatorname{Im}\left(A_{\perp}^{L}\right), \operatorname{Re}\left(A_{\|}^{L}\right), \operatorname{Im}\left(A_{\|}^{L}\right), \operatorname{Re}\left(A_{0}^{L}\right), \operatorname{Im}\left(A_{0}^{L}\right),\right. \\
& \left.\operatorname{Re}\left(A_{\perp}^{R}\right), \operatorname{Im}\left(A_{\perp}^{R}\right), \operatorname{Re}\left(A_{\|}^{R}\right), \operatorname{Im}\left(A_{\|}^{R}\right), \operatorname{Re}\left(A_{0}^{R}\right), \operatorname{Im}\left(A_{0}^{R}\right)\right) .
\end{aligned}
$$

$\vec{S}$ represents a symmetry of the distribution if and only if $\forall i \in\left(J_{1 s} \ldots J_{9}\right): \vec{\nabla}\left(J_{i}\right) \perp \vec{S}$.

There are as many independent infinitesimal symmetries as linearly independent vectors $\vec{S}_{j}$, with $j=1, . . n$ satisfying the above constraint. In the case of massless leptons with no scalars four of those vectors $\vec{S}_{j}$ were found [10]. This is the first proof that four and no more symmetries are present.

The explicit form of the four continuous independent symmetry transformations ${ }^{2}$ of the amplitudes that leave the differential distribution invariant are [10]:

$$
n_{i}^{\prime}=\left[\begin{array}{ll}
e^{i \phi_{L}} & 0 \\
0 & e^{-i \phi_{R}}
\end{array}\right]\left[\begin{array}{rr}
\cos \theta & -\sin \theta \\
\sin \theta & \cos \theta
\end{array}\right]\left[\begin{array}{rr}
\cosh i \tilde{\theta} & -\sinh i \tilde{\theta} \\
-\sinh i \tilde{\theta} & \cosh i \tilde{\theta}
\end{array}\right] n_{i}
$$

\footnotetext{
${ }^{2}$ Sometimes it might be a non-trivial task to find a continuous symmetry associated to an infinitesimal one.
} 


$$
\begin{array}{cc}
A_{\perp}^{L}=\left[\frac{\frac{4}{9} J_{1 s}^{2}-J_{3}^{2}-\frac{1}{4} J_{6 s}^{2}-J_{9}^{2}}{\frac{2}{3} J_{1 s}-J_{3}}\right]^{\frac{1}{2}} e^{i \phi_{\perp}^{L}} & A_{\perp}^{R}=-\frac{\left(J_{6 s}-2 i J_{9}\right)}{2 \sqrt{\frac{2}{3} J_{1 s}-J_{3}}} \\
A_{\|}^{L}=0 & A_{\|}^{R}=\sqrt{\frac{2}{3} J_{1 s}-J_{3}} \\
A_{0}^{L}=\left[\frac{J_{1 c}\left(\frac{2}{3} J_{1 s}-J_{3}\right)-2 J_{4}^{2}-\frac{1}{2} J_{7}^{2}}{\frac{2}{3} J_{1 s}-J_{3}}\right]^{\frac{1}{2}} e^{i \phi_{0}^{L}} & A_{0}^{R}=\frac{2 J_{4}-i J_{7}}{\sqrt{\frac{4}{3} J_{1 s}-2 J_{3}}}
\end{array}
$$

Table 1: Explicit solution of the spin amplitudes in terms of the coefficients of the distribution for the massless case without scalars.

where we have defined $n_{1}=\left(A_{\|}^{L}, A_{\|}^{R^{*}}\right), n_{2}=\left(A_{\perp}^{L},-A_{\perp}^{R^{*}}\right)$ and $n_{3}=\left(A_{0}^{L}, A_{0}^{R^{*}}\right)$. The first two symmetries (phase transformations) are a consequence of the freedom to pick up an arbritrary and different global phase for the $\mathrm{L}$ and $\mathrm{R}$ non-interfering amplitudes. The third and fourth symmetry corresponds to the experimental impossibility to measure a simultaneous change of helicity and handedness of the current (a helicity +1 state with a left handed current transforms into a helicity -1 with a right handed current).

But, what have we learnt from using this symmetry approach? The answer to this question is twofold. On the one side, it basically gives freedom to construct an optimal observable out of the spin amplitudes. The symmetries allow to bypass the strong restriction of taking each coefficient of the distribution as an observable and permits to construct the best, i.e. most sensitive to NP, combination of them. The only requirement to fulfil is that the constructed quantity has to respect these symmetries (in order to be promoted to an observable). On the other side, the symmetries of the distribution are necessary to find a solution of the system of the spin amplitudes in terms of the coefficients of the distribution; in particular it allows us to identify new hidden correlations which turn out to be important for the stability of the experimental fit.

Indeed we found in Ref.[10] that all the physical information of the distribution is encoded in the three moduli and the complex scalar products of the vectors $n_{i}$,

$$
\begin{aligned}
\left|n_{1}\right|^{2} & =\frac{2}{3} J_{1 s}-J_{3}, & \left|n_{2}\right|^{2} & =\frac{2}{3} J_{1 s}+J_{3}, \\
n_{1} \cdot n_{2} & =\frac{J_{6 s}}{2}-i J_{9}, & n_{1} \cdot n_{3} & =\sqrt{2} J_{4}-i \frac{J_{7}}{\sqrt{2}},
\end{aligned}
$$

The symmetries guarantee the invariance of these moduli and scalar products. Using the freedom given by the symmetries to fix certain parameters to zero, the system of $A$ 's can be solved in terms of $J$ 's. In particular, we choose the left global phase $\left(\phi_{L}\right)$ such that $\operatorname{Im} A_{\|}^{L}=0$, the right global phase symmetry $\left(\phi_{R}\right)$ such that $\operatorname{Im} A_{\|}^{R}=0$ (simplicity) and one of the continuous $L \leftrightarrow R$ rotation $\theta$ to fix $\operatorname{Re} A_{\|}^{R}=0$. The system is then easily solved as shown in Table 1 . Still one last equation remains

$$
e^{i\left(\phi_{\perp}^{L}-\phi_{0}^{L}\right)}=\frac{J_{5}\left(\frac{2}{3} J_{1 s}-J_{3}\right)-J_{4} J_{6 s}-J_{7} J_{9}-i\left(\frac{4}{3} J_{1 s} J_{8}-2 J_{3} J_{8}+2 J_{4} J_{9}-\frac{1}{2} J_{6 s} J_{7}\right)}{\left[2\left(\frac{4}{9} J_{1 s}^{2}-J_{3}^{2}-\frac{1}{4} J_{6 s}^{2}-J_{9}^{2}\right)\left(J_{1 c}\left(\frac{2}{3} J_{1 s}-J_{3}\right)-2 J_{4}^{2}-\frac{1}{2} J_{7}^{2}\right)\right]^{1 / 2}} .
$$

This equation has two important consequences. First, it represents another proof of the existence of the fourth symmetry manifesting itself in the freedom to choose either $\phi_{\perp}^{L}$ or $\phi_{0}^{L}=0$. And second, the condition of the LHS of this equation being a phase impose the following non-trivial constrain on the RHS:

$$
J_{1 c}=-J_{2 c}=6 \frac{\left(2 J_{1 s}+3 J_{3}\right)\left(4 J_{4}^{2}+J_{7}^{2}\right)+\left(2 J_{1 s}-3 J_{3}\right)\left(J_{5}^{2}+4 J_{8}^{2}\right)}{16 J_{1 s}^{2}-9\left(4 J_{3}^{2}+J_{6 s}^{2}+4 J_{9}^{2}\right)}
$$




$$
-36 \frac{J_{65}\left(J_{4} J_{5}+J_{7} J_{8}\right)+J_{9}\left(J_{5} J_{7}-4 J_{4} J_{8}\right)}{16 J_{1 s}^{2}-9\left(4 J_{3}^{2}+J_{6 s}^{2}+4 J_{9}^{2}\right)} \equiv f .
$$

We emphasize that this equation holds in the case without scalar amplitudes and under the assumption that the mass of the leptons can be neglected. If scalar amplitudes are relevant $\left(J_{1 c} \neq-J_{2 c}\right)$ the equation $-J_{2 c}=f$ is still fulfilled while $J_{1 c} \neq f$. Taking into account the mass of the leptons, one can derive a similar expression only if there are no scalar amplitudes included in the analysis. As a consequence, if $J_{1 c}=f$ is not fulfilled and large deviations are observed (small deviations may be due to the massive terms) this would signal the presence of scalars. On the contrary, if the equation $-J_{2 c}=f$ is not fulfilled and large deviations are observed it might point to an experimental problem.

\section{Construction of transverse observables: $A_{T}^{(i)}$}

Following the previous steps we constructed four different robust observables. Two of them $A_{T}^{(2)}$ and $A_{T}^{(5)}$ are only sensitive to the transverse amplitudes, while $A_{T}^{(3)}$ and $A_{T}^{(4)}$ also have sensitivity to the longitudinal spin amplitude. In this section we will focus on the properties of the former. The computation of spin amplitudes $A_{\perp, \|, 0}^{(L, R)}$ is done at the NLO level within the framework of QCD-factorization [3] . They are functions of the long-distance $B \rightarrow K^{*}$ form factors $\left(A_{0,1,2}\left(q^{2}\right), V\left(q^{2}\right), T_{1,2,3}\left(q^{2}\right)\right)$ (see Ref.A [13] for a recent analysis) and of the short-distance Wilson coefficients $\left(C_{7}^{\text {eff }}, C_{7}^{\text {eff' }}, C_{9}^{\text {eff }}, C_{10}, C_{9}^{\text {eff' }}, C_{10}^{\prime}\right.$ ) (for precise definitions see Refs. [9, 10]). In the heavy quark and large $E_{K^{*}}$ limit all form factors can be expressed in terms of just two soft form factors $\xi_{\perp}\left(E_{K}^{*}\right)$ and $\xi_{\|}\left(E_{K}^{*}\right)$ [14]. However these relations receive two types of corrections: order $\alpha_{s}[3,6]$ (coming from NLO-QCDf) and power suppressed $\Lambda / m_{b}$ corrections estimated to be of $\mathscr{O}(10 \%)$. Both were included in our computation of the spin amplitudes at the NLO level in Refs. $[9,10]$.
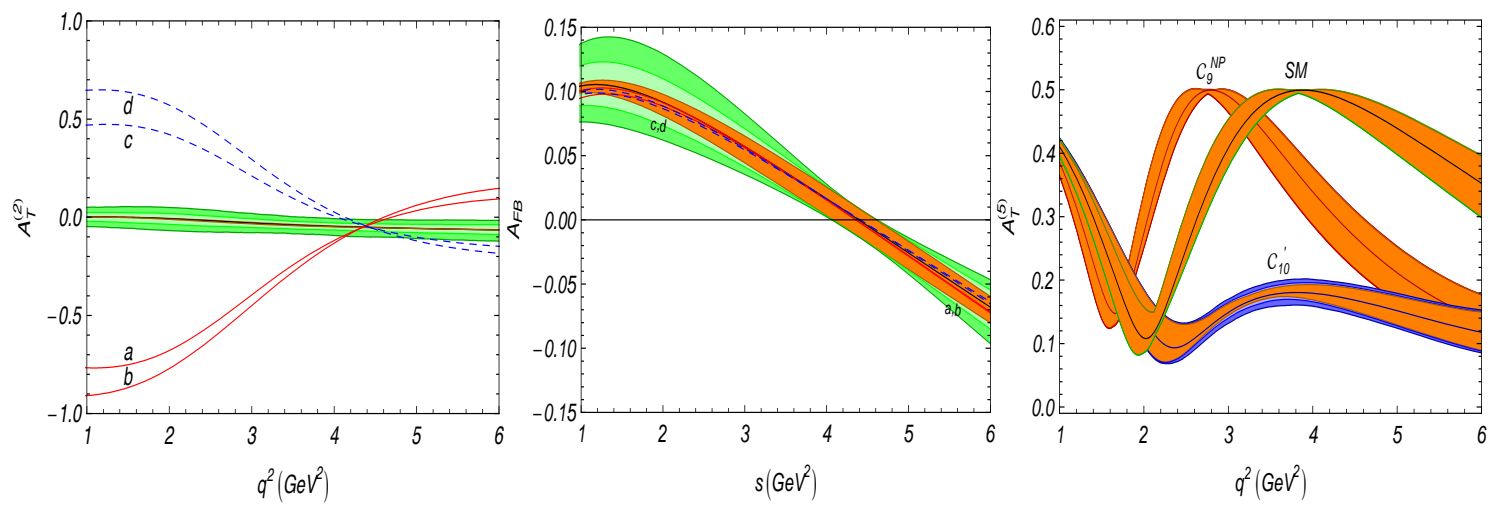

Figure 1: Left: $A_{T}^{2}$ in SM (green band) with four NP benchmarks ([10]). Center: $A_{F B}$ for the same cases. Right: $A_{T}^{5}$ in the SM and for different values of $C_{9}^{e f f}$ and $C_{10}^{\prime}$ (for more details see Ref.[10]).

The observable $A_{T}^{(2)}$ was first proposed in Ref. [7]:

$$
A_{T}^{2}=\frac{\left|A_{\perp}\right|^{2}-\left|A_{\|}\right|^{2}}{\left|A_{\perp}\right|^{2}+\left|A_{\|}\right|^{2}} .
$$


It is built to signal deviations from the left-handed structure of the SM: $\left.A_{T}^{2}\right|_{S M} \sim 0$. We restricted our analysis to the $1<q^{2}<6 \mathrm{GeV}^{2}$ region (its extension to $q^{2}>14 \mathrm{GeV}^{2}$ was described in [15]). Some of the most important properties are:

- The soft form factor dependence cancels exactly at LO and a very mild dependence at NLO is observed.

- In the large recoil limit for the spin amplitudes $A_{T}^{(2)}$ simplifies to

$$
A_{T}^{2} \sim 4 C_{7}^{\mathrm{eff}} \frac{m_{b} M_{B}}{s} \frac{\Delta_{-}+\Delta_{+}^{*}}{2 C_{10}^{2}+\left|\Delta_{-}\right|^{2}+\left|\Delta_{+}\right|^{2}}
$$

where $\Delta_{ \pm}=\mathscr{C}_{9}^{\text {eff }}+2 \frac{m_{b} M_{B}}{s}\left(C_{7}^{\text {eff }} \pm C_{7}^{\text {eff' }}\right)$. The strong sensitivity to the coefficient $C_{7}^{\text {eff' }}$ of the electromagnetic chirally flipped operator and an important enhancement factor $2 m_{b} M_{B} / s$ around $1 \mathrm{GeV}^{2}$ are evident.

- The comparison between $A_{T}^{(2)}$ and $A_{F B}$ is particularly interesting: i) While $A_{T}^{(2)}$ is extremely sensitive to right-handed currents via $C_{7}^{\prime}$ (and its CP violating phase), $A_{F B}$ shows only a very mild (for the modulus) or null (for the phase) sensitivity (see also Fig. 1, right and center plot). ii) Both observables exhibit a zero, or a lack of it, at the same value of $q_{0}^{2}$ at LO (but also at NLO) if $C_{7}^{\text {eff' }} \neq 0$. iii) While $A_{F B}$ is only protected from large soft form factor uncertainties at its zero, $A_{T}^{(2)}$ is protected in the whole $1<q^{2}<6 \mathrm{GeV}^{2}$ region.

- $A_{T}^{(2)}$ also serves as an excellent probe for a nontrivial $C_{10}^{\prime}$. The latter implies a completely different $q^{2}$ dependence than a non-zero coefficient $C_{7}^{\prime}$.

- $A_{T}^{(2)}$ can be measured using the one-angle projected angular distribution in the first run of data taking with the LHCb experiment and using the full angular distribution afterwards. See $[9,10]$ for a discussion of its experimental sensitivity.

The transverse observable $A_{T}^{(5)}$, complementary to $A_{T}^{(2)}$, was proposed in Ref. [10]:

$$
A_{T}^{(5)}=\frac{\left|A_{\|}^{R *} A_{\perp}^{L}+A_{\|}^{L} A_{\perp}^{R *}\right|}{\left|A_{\|}\right|^{2}+\left|A_{\perp}\right|^{2}} .
$$

- Contrary to $A_{T}^{(2)}, A_{T}^{(5)}$ exhibits a combination of left-right and $\perp$-\| amplitudes that cannot be found in any single coefficient of the distribution. Its expression in terms of the coefficients of the distribution can be found using the explicit solution described in Sec.2:

$$
\left.A_{T}^{5}\right|_{m_{\ell}=0}=\frac{\sqrt{16 J_{1}^{s 2}-9 J_{6}^{s 2}-36\left(J_{3}^{2}+J_{9}^{2}\right)}}{8 J_{1}^{s}} .
$$

- In the large recoil limit and assuming a nontrivial $C_{10}^{\prime}, A_{T}^{(5)}$ simplifies to

$$
\left.A_{T}^{(5)}\right|_{10^{\prime}}=\frac{\left.\left|-C_{10}^{2}+\right| C_{10}^{\prime}\right|^{2}+\left(2 m_{b} M_{B} C_{7}^{\mathrm{eff}} / q^{2}+C_{9}^{\mathrm{eff}}\right)^{2} \mid}{2\left[C_{10}^{2}+\left|C_{10}^{\prime}\right|^{2}+\left(2 m_{b} M_{B} C_{7}^{\mathrm{eff}} / q^{2}+C_{9}^{\mathrm{eff}}\right)^{2}\right]} .
$$


It implies that $A_{T}^{(5)}$ has a maximum value in the $\mathrm{SM}\left(C_{10}^{\prime}=0\right)$ of $1 / 2$ near to the position of the zero of $A_{F B}$. If $C_{10}^{\prime} \neq 0$ and $C_{10}^{\prime}<C_{10}$ the size of the local maximum decreases and its distance to the SM maximum is given by $\left|C_{10}^{\prime N P}\right|^{2} /\left(C_{10}^{2}+\left|C_{10}^{\prime N P}\right|^{2}\right)$. This distance can be used as a measurement of $C_{10}^{\prime}$ if $C_{10}^{\prime}$ represents the only contribution beyond the SM (see Fig.1, left plot).

- Finally, the position $q_{0}^{2}$ of the maximum moves if $C_{7}^{\text {eff }}$ or $C_{9}^{\text {eff }}$ receives NP contributions like $A_{F B}$ (see again Fig.1, left plot).

\section{Conclusions}

We have presented in detail a method to construct observables, using the $K^{*}$ spin amplitudes as building blocks, with high new physics sensitivity and reduced hadronic pollution. It is sufficiently general to be applied to other angular decays with similar properties. The symmetries of the fourbody decay, that play a central role in this method, are identified and interpreted. Finally, two observables are constructed fulfilling all the steps of the method and their properties are analyzed. $A_{T}^{2}$ emerges as an improved version of $A_{F B}$, containing almost all the physical information of it but in a less QCD polluted way, and it also exhibits a much larger sensitivity to right-handed currents than $A_{F B}$.

\section{References}

[1] T. Hurth, M. Nakao, Ann. Rev. Nucl. Part. Sci. 60 (2010) 645. [arXiv:1005.1224 [hep-ph]].

[2] G. Burdman, Phys. Rev. D 57 (1998) 4254. [arXiv:hep-ph/9710550].

[3] M. Beneke, T. Feldmann, D. Seidel, Nucl. Phys. B 612 (2001) 25. [arXiv:hep-ph/0106067];

[4] A. K. Alok et al., JHEP 1002 (2010) 053. [arXiv:0912.1382 [hep-ph]].

[5] T. Feldmann and J. Matias, JHEP 0301 (2003) 074 [arXiv:hep-ph/0212158].

[6] M. Beneke, T. Feldmann, D. Seidel, Eur. Phys. J. C 41 (2005) 173. [arXiv:hep-ph/0412400].

[7] F. Kruger and J. Matias, Phys. Rev. D 71 (2005) 094009 [arXiv:hep-ph/0502060].

[8] E. Lunghi and J. Matias, JHEP 0704 (2007) 058 [arXiv:hep-ph/0612166].

[9] U. Egede, T. Hurth, J. Matias, M. Ramon, W. Reece, JHEP 0811 (2008) 032. [arXiv:0807.2589 [hep-ph]].

[10] U. Egede, T. Hurth, J. Matias, M. Ramon, W. Reece, JHEP 1010 (2010) 056. [arXiv:1005.0571 [hep-ph]].

[11] W. Altmannshofer, P. Ball, A. Bharucha, A. J. Buras, D. M. Straub, M. Wick, JHEP 0901 (2009) 019. [arXiv:0811.1214 [hep-ph]].

[12] E. Lunghi, A. Soni, JHEP 1011 (2010) 121. [arXiv:1007.4015 [hep-ph]].

[13] A. Khodjamirian, T. Mannel, A. A. Pivovarov, Y. M. Wang, JHEP 1009 (2010) 089. [arXiv:1006.4945 [hep-ph]].

[14] J. Charles, A. Le Yaouanc, L. Oliver, O. Pene, J. C. Raynal, Phys. Rev. D 60 (1999) 014001. [arXiv:hep-ph/9812358].

[15] C. Bobeth, G. Hiller, D. van Dyk, JHEP 1007 (2010) 098. [arXiv:1006.5013 [hep-ph]]. 